\title{
Modélisation statistique de la détresse psychologique chez les personnes âgées vivant en milieu rural et urbain
}

\author{
C.P. Karunanayake, Ph.D. (1); P. Pahwa, Ph.D. $(1,2)$
}

\section{Résumé}

L'augmentation rapide du nombre de personnes âgées au Canada entraînera une augmentation de la demande de soins de santé chez les aînés qui souffrent de troubles mentaux. Comme les personnes âgées sont plus nombreuses à vivre en zone rurale que les plus jeunes, il est important de cerner les différences entre les personnes âgées vivant en milieu rural et celles vivant en milieu urbain afin de concevoir et de fournir des soins de santé mentale appropriés. L’objectif principal de cette étude était d'utiliser l'Enquête nationale sur la santé de la population (ENSP) afin d'examiner les différences à l'égard de la détresse psychologique entre les personnes âgées vivant en milieu rural et celles vivant en milieu urbain (il s'agit des personnes de plus de 55 ans). Les autres objectifs consistaient à examiner l'association à long terme entre l'usage du tabac et la santé mentale et l'association à long terme entre les besoins de soins de santé non satisfaits et la santé mentale des personnes âgées vivant en milieu rural et en milieu urbain. La mesure de la détresse psychologique a été examinée comme un résultat binaire. L’analyse a été menée selon le modèle de l'équation d'estimation généralisée qui a pris en compte la complexité d'un plan de sondage à plusieurs degrés. Les personnes âgées vivant en milieu rural étaient proportionnellement plus nombreuses à déclarer une détresse psychologique que les personnes âgées vivant en milieu urbain. Cette incidence était toutefois à la limite de la signification statistique [RC $=1,16$; IC à $95 \%: 0,98,1,37]$. Cette conclusion s'est fondée sur un modèle final à variables multiples qui avait pour objet de déterminer s'il existe un lien entre la détresse psychologique et le lieu de résidence (rural ou urbain) de même qu'entre l'usage du tabac et l'autodéclaration de besoins de soins de santé non satisfaits. Ce modèle a été ajusté afin de prendre en compte d'autres covariables importantes et le manque de données relatives à la variable étudiée. Une corrélation significative a été notée entre l'usage du tabac et les problèmes de santé mentale chez les personnes âgées après que l'on a effectué des ajustements pour prendre en compte d'autres covariables [RC $=1,26$; IC à $95 \%: 1,00,1,60]$. Les participants ayant déclaré des besoins de soins de santé non satisfaits étaient proportionnellement plus nombreux à déclarer une détresse psychologique $[\mathrm{RC}=1,72$; IC à $95 \%: 1,38,2,13]$ que ceux qui se disaient satisfaits de leurs soins de santé.

\section{Mots clés : santé mentale, personnes âgées vivant en milieu rural, données longitudinales, Étude nationale sur la santé de la population, équations d'estimation généralisées, les poids bootstrap, données manquantes}

\section{Introduction}

Les personnes âgées forment le groupe de population qui connaît la croissance la plus rapide au Canada tel que le rapporte
Statistique Canada. En 2021, il y aura presque sept millions de personnes âgées (65 ans et plus), et elles représenteront $19 \%$ de la population ${ }^{1}$. Les personnes âgées sont plus nombreuses à vivre en milieu rural que les plus jeunes (24\% contre $21 \%$ ) et à vivre dans de plus petits milieux urbains $^{1}$. Les personnes âgées des milieux ruraux vivent souvent dans l'isolement et présentent ainsi un risque plus élevé de développer des problèmes de santé mentale que leurs pairs vivant en milieu urbain en raison d'une interaction sociale réduite. En outre, cet isolement peut accroître chez les personnes âgées vivant en milieu rural la possibilité d'adopter des habitudes de vie, tels l'usage du tabac et la consommation d'alcool, qui contribuent à l'aggravation des problèmes de santé mentale ${ }^{2-4}$. Les maladies mentales interviennent pour $30 \%$ dans les demandes d'indemnité pour invalidité, soit de 15 à 33 milliards de dollars par année au Canada ${ }^{5}$. Une étude canadienne récente évalue le coût annuel des maladies mentales traitées et non traitées au Canada à 14,4 milliards de dollars ${ }^{6}$. En 2020, la dépression sera la deuxième maladie d'importance mondiale derrière les maladies ischémiques ${ }^{7-8}$. Les progrès de la neuroscience et de la médecine comportementale ont établi que les troubles mentaux sont causés par des interactions complexes dans lesquelles interviennent des facteurs biologiques, psychologiques et sociaux $^{8}$. Il y a eu suffisamment d'études portant sur la santé mentale des personnes âgées vivant en milieu rural, mais très peu d'études comparatives entre les personnes âgées vivant en milieu rural et celles vivant en milieu urbain. Il est important de cerner les différences entre les personnes âgées vivant en milieu rural et celles vivant en milieu urbain afin de concevoir et de fournir des soins de santé mentale

Coordonnées des auteurs

1 Centre canadien de santé et sécurité en milieu agricole, Université de la Saskatchewan Saskatoon (Saskatchewan)

2 Département de santé communautaire et d'épidémiologie, Université de la Saskatchewan, Saskatoon (Saskatchewan)

Correspondance : Chandima Karunanayake, Ph.D., Centre canadien de santé et sécurité en milieu agricole, Université de la Saskatchewan, Royal University Hospital, 103 Hospital Drive, Saskatoon (Saskatchewan) S7N 0W8, Tél. : 306-966-1647, Téléc. : 306-966-8799, Courriel : cpk646@mail.usask.ca 
appropriés. L’analyse statistique appropriée des ensembles de données longitudinales nationales disponibles permet d'étudier les facteurs de risque importants de la détresse psychologique. Ces facteurs peuvent nous conduire à identifier les groupes présentant un risque élevé à un stade précoce et nous aider à cibler nos mesures préventives de manière à réduire l'incidence économique que la dépression aura sur le système de santé dans les années qui viennent ${ }^{9}$.

Les auteurs de la présente étude se sont servis de l'Enquête nationale sur la santé de la population (ENSP) ${ }^{10}$ de Statistique Canada pour examiner (1) les différences entre le milieu rural et le milieu urbain en ce qui a trait à la détresse psychologique, (2) l'association à long terme entre l'usage du tabac et la détresse psychologique, et (3) l'association à long terme entre l'insuffisance de soins de santé et la santé mentale des personnes âgées vivant en milieu rural et celles vivant en milieu urbain. Le milieu urbain se définit comme une zone bâtie en continu ayant une population de 1000 personnes ou plus et une densité de population d'au moins 400 personnes par kilomètre carré, selon le recensement précédent ${ }^{11}$; les autres régions sont définies comme des zones rurales.

\section{Méthodes}

\section{Base de données longitudinales de l'ENSP}

Les données de l'Enquête nationale sur la santé de la population (ENSP) ont été utilisées pour cette analyse. L'ENSP est une étude longitudinale ${ }^{10}$ d'un échantillon national canadien. L'enquête comptait initialement 17626 sujets échantillonnés en 1994-1995 (soit le cycle 1) dans le but de les suivre ou de les recontacter tous les deux ans pendant une période pouvant durer 20 ans. Afin de pouvoir participer à l'étude, les répondants doivent avoir complété au moins la partie générale du questionnaire en 1994-1995'12. L'ENPS a eu recours, sauf dans la province de Québec, à un plan d'échantillonnage stratifié à deux degrés (grappes, logements) fondé sur celui de l'Enquête sur la population active (EPA) de Statistique Canada. Au Québec, l'échantillon de l'ENSP a été prélevé à partir d'un plan à deux degrés semblable à celui de l'EPA employé pour les logements qui ont participé à l'Enquête sociale et de santé (ESS) réalisée par Santé Québec en 1992-1993'13. La taille des échantillons de base de chaque province a été établie selon la méthode de répartition de Kish, qui a permis d'établir un juste équilibre entre les exigences de fiabilité aux niveaux national et provincial. Il fallait au moins 1200 ménages par province pour produire des données suffisamment fiables selon le sexe et les grands groupes d'âge. Les populations vivant dans les réserves des Premières nations, les bases des Forces canadiennes et certaines régions éloignées du Québec et de l'Ontario ont été exclues de la composante des ménages de l'enquête. Les données ont été pondérées afin de tenir compte du plan d'échantillonnage, de la correction pour la non-réponse et de la stratification a posteriori.

Les mêmes individus ont été sondés à plusieurs reprises, ce qui a permis d'étudier l'incidence des facteurs de référence et des facteurs de risque variables dans le temps sur l'évolution de l'état de santé mentale. Les conclusions qui découlent de telles enquêtes longitudinales sont plus rigoureuses que celles tirées des enquêtes transversales, étant donné qu'il existe de l'information sur la séquence d'événements $^{14}$. Dans la plupart des cas, les données longitudinales sont incomplètes. Il existe plusieurs façons d'analyser les données longitudinales incomplètes. Un modèle de régression logistique binaire peut être employé si l'on recourt à une méthode fondée sur l'improbabilité, à savoir le modèle de l'équation d'estimation généralisée (EEG) ${ }^{15}$, en supposant que les données ne manquent pas complètement au hasard $(\mathrm{MCAH})$. Une autre façon d'analyser les données longitudinales incomplètes consiste à utiliser des modèles de mélange d'observation de schémas formulés par Little ${ }^{16-18}$, en supposant que les données manquantes ne sont pas MCAH. Le modèle de mélange de schémas est une solution au problème de la non-réponse dans les données d'enquête. La première étape de l'application du modèle de mélange de schémas consiste à diviser les sujets en groupes en fonction de leur schéma de données manquantes. Si les sujets sont sondés à six moments différents dans le temps, il y aura alors $64\left(2^{6}\right)$ schémas possibles de données manquantes. Une variable inter-sujets est créée en groupant les schémas de données manquantes. Cette variable inter-sujets peut aussi être employée comme covariable dans l'analyse des données longitudinales. La présente étude examine des modèles de mélange de schémas à l'aide de modèles fondés sur l'EEG qui ont servi dans l'Enquête nationale sur la santé de la population.

\section{Échelle de détresse : enquête nationale sur la santé de la population (ENSP)}

De l'éventail relativement vaste des indicateurs de maladie mentale recensés dans l'ENSP, nous avons choisi de mesurer la détresse en nous fondant sur un sous-ensemble d'items tirés du Composite International Diagnostic Interview (CIDI). Le point d'intérêt consistait en six questions mises au point par Kessler et Mroczek de l'University of Michigan ${ }^{19}$. L'échelle de détresse se compose de plusieurs questions tirées du CIDI qui portent sur la tristesse, la nervosité, l'inquiétude, le désespoir, la dévalorisation de soi et l'impression que tout est difficile ${ }^{11}$. D'autres questions permettaient de déterminer si ces sentiments survenaient " souvent ", " quelque peu ", " un peu », «plus que d'habitude », «pas de changement » ou " moins souvent que d'habitude » par rapport au mois précédent. En se fondant sur ces questions, une échelle de détresse a été dérivée pour chacun des six cycles. Cette variable dérivée sert à situer le répondant sur l'échelle de détresse. Les scores sur l'échelle de détresse vont de 0 (pas de détresse) à 24 (très haut degré de détresse). Les précisions sont disponibles dans le répertoire de l'ENSP sur la variable dérivée ${ }^{11}$.

\section{Sous-population}

L'étude initiale effectuée en 1994-1995 était limitée à la proportion de la population âgée de 55 ans et plus. La sous-population comptait 4444 participants et l'analyse longitudinale comptait 16052 observations. Les principaux facteurs étudiés sont le lieu de résidence et l'usage du tabac et de l'alcool. Les autres variables démographiques et socioéconomiques provenant d'études antérieures sur la santé mentale ${ }^{20-23}$ qui sont incluses dans ces analyses sont le sexe, l'âge, la situation de famille, le niveau de scolarité, le revenu familial, l'indice de 
santé générale autodéclaré, la zone géographique, l'existence de conditions chroniques, l'activité physique au cours des trois derniers mois et les besoins non satisfaits de soins de santé.

\section{Modélisation de la détresse comme variable binaire}

Il a été intéressant de suivre l'évolution du vecteur de réponse dans le temps et d'examiner comment il est associé à un ensemble de variables explicatives. L'échelle de détresse présentait une importante asymétrie et nous avons décidé de la recoder en nous appuyant sur la documentation ${ }^{23-24}$ et sur les suggestions d'un gérontopsychiatre. Étant donné que la détresse a été recodée en fonction d'une échelle binaire (catégories : nonfaible [échelle 0-5] et modérée-élevée [échelle 6-24]), il est apparu naturel d'employer un modèle binaire. Nous avons ajusté un modèle de régression binaire fondé sur l'EEG ${ }^{25-26}$ à l'aide de la procédure GENMOD de $\mathrm{SAS}^{25-29}$. Cette procédure nous a permis de choisir différentes spécifications de matrices de corrélation temporaires (indépendante, autorégressive de premier ordre $[\mathrm{AR}(1)]$, interchangeable et non structurée ). Nous avons choisi le modèle qui possède une structure de covariance quelconque afin d'obtenir les erreurs-types les plus faibles ${ }^{30}$. La GENMOD est fondée sur la méthode de Liang et Zeger ${ }^{28-29}$, qui ne tient compte que des corrélations intra-sujet en raison de la répétition des mesures dans le temps. Afin de tenir compte de la complexité du plan d'échantillonnage à plusieurs degrés stratifié, la méthode de rééchantillonnage bootstrap a été utilisée afin d'obtenir un calcul précis de la variance d'une estimation donnée. Ces résultats ont été obtenus grâce au programme de macros Bootvar (en format SAS) ${ }^{30-32}$ et à l'aide des poids bootstrap fournis par Satitistique Canada ${ }^{10}{ }^{12}$. Le programme de macros Bootvar a été modifié de manière à s'appliquer à la méthode d'estimation des équations généralisées ${ }^{31,34}$. Le modèle statistique employé pour étudier l'effet des données manquantes est le modèle de mélange de schémas fondé sur l'EEG.

\section{Analyse statistique}

Des analyses à une variable ont été menées afin d'examiner le lien entre l'échelle de détresse et les principaux facteurs considérés, de même que les variables démographiques et socioéconomiques au niveau de signification $\alpha=0,20$. L'étape suivante a consisté en une analyse multidimensionnelle visant à déterminer les effets de toutes les covariables possibles ou interactions sur l'échelle de détresse. Toutes les covariables possibles et les termes d'interaction ont été inclus simultanément dans le modèle. Les variables qui étaient significatives au niveau $\alpha=0,05$ ou qui présentaient un intérêt scientifique, de même que les schémas de données manquantes ont été retenus dans le modèle final.

\section{Résultats}

En 1994, les personnes âgées représentaient $20 \%$ de la population totale du Canada. Les femmes représentaient $56 \%$ des personnes âgées. En milieu rural, $22 \%$ des résidents étaient des personnes âgées tandis qu'en milieu urbain les personnes âgées représentaient $20 \%$ de la population. En milieu rural, les hommes représentaient $53 \%$ des personnes âgées tandis que les femmes représentaient $58 \%$ des personnes âgées en milieu urbain. L'objet principal de cette analyse était de comparer le degré de détresse psychologique des personnes âgées vivant en milieu rural et de celles vivant en milieu urbain. Nous avons commencé l'analyse statistique en explorant les différences dans les caractéristiques de base présentées au Tableau 1 et résumées ci-dessous.

\section{Comparaison du degré de détresse psychologique des personnes âgées vivant en milieu rural et de celles vivant en milieu urbain}

Dix-sept pourcent des personnes âgées vivant en milieu rural et $16 \%$ de celles vivant en milieu urbain se retrouvaient dans la catégorie des personnes présentant une détresse modérée ou élevée. Par groupe d'âge la proportion des personnes ayant un degré de détresse psychologique modéré ou élevé varie de 12 à $25 \%$ pour les personnes âgées vivant en milieu rural et de 14 à $19 \%$ pour les personnes âgées vivant en milieu urbain. Dans la catégorie des personnes présentant une détresse modérée ou élevée, il y avait un pourcentage légèrement plus élevé (22\%) de femmes âgées vivant en milieu rural que de femmes âgées vivant en milieu urbain (20\%). De plus, les femmes âgées avaient un niveau de détresse plus élevé que les hommes âgés et ce tant en milieu rural qu'en milieu urbain. Les personnes âgées vivant en milieu rural qui étaient célibataires, mariées, conjoints de fait ou vivaient avec un partenaire étaient proportionnellement plus nombreuses à présenter un niveau de détresse modéré ou élevé que leurs homologues vivant en milieu urbain. $\mathrm{Au}$ Québec, tant les personnes vivant en milieu rural que celles vivant en milieu urbain étaient proportionnellement plus nombreuses à présenter un niveau de détresse modéré ou élevé que les personnes âgées vivant en milieu rural ou urbain dans d'autres régions du Canada. Dans les zones rurales et urbaines, les répondants ayant un faible niveau de scolarité étaient proportionnellement plus nombreux à présenter un niveau de détresse modéré ou élevé que les personnes ayant complété des études postsecondaires et la proportion des répondants à faible revenu se retrouvant dans la catégorie de la détresse modérée ou élevée était aussi plus élevée que la proportion correspondante des répondants ayant un revenu élevé. De plus, la proportion des personnes âgées ayant peu d'activités sociales et qui faisaient état d'une détresse modérée ou élevée était plus forte tant en milieu rural qu'en milieu urbain. Les personnes âgées qui fumaient étaient proportionnellement plus nombreuses à présenter un niveau de détresse modéré ou élevé que les personnes âgées qui ne fumaient pas, et ce tant en milieu rural qu'en milieu urbain. De même, les personnes qui ne consommaient pas d'alcool étaient proportionnellement plus nombreuses (21\%) à présenter un degré de détresse modéré ou élevé que les personnes qui consommaient de l'alcool, et ce tant en milieu rural qu'en milieu urbain.

Enfin, les répondants qui souffraient d'une condition chronique étaient plus exposés au risque de détresse psychologique modéré ou élevé que ceux ne faisant pas état d'une condition chronique; il en était de même pour les répondants qui n’avaient pratiqué aucune activité physique au cours des trois derniers mois comparativement à ceux qui avaient pratiqué des activités physiques. 
FIGURE 1

Autodéclaration de besoins de soins de santé non satisfaits des personnes âgées vivant en milieu rural et en milieu urbain au fil du temps

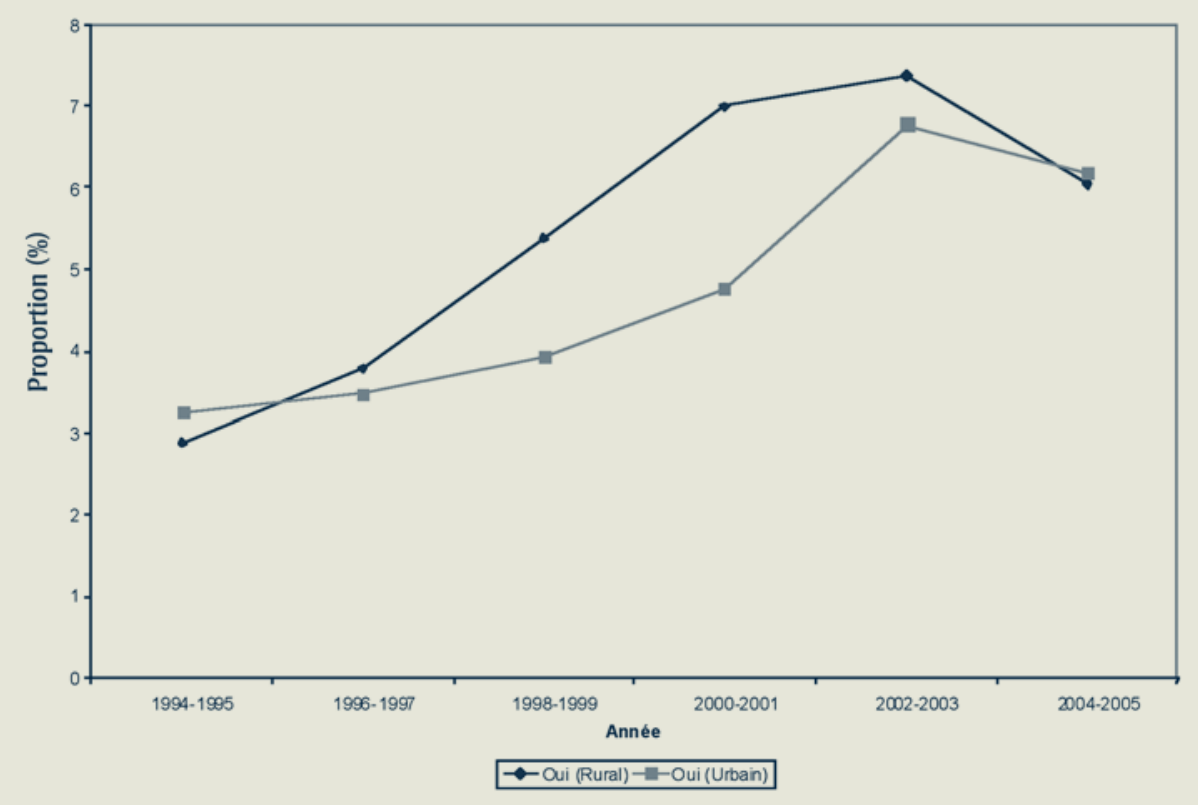

De plus, les personnes âgées qui avaient déclaré des besoins de soins de santé non satisfaits étaient proportionnellement plus nombreuses à subir un niveau de détresse modéré ou élevé que celles qui avaient l'impression que leurs besoins de soins de santé étaient satisfaits. Cette proportion était plus élevée en milieu urbain, $49 \%$, qu'en milieu rural, $43 \%$.

La figure 1 illustre la comparaison entre le milieu rural et le milieu urbain en ce qui a trait à l'autodéclaration de besoins de soins de santé non satisfaits. Cette figure laisse supposer que les personnes âgées vivant en milieu rural avaient plus de chances d'avoir des besoins de santé non satisfaits que leurs homologues vivant en milieu urbain de 1996 à 2002. Cette augmentation apparente dans le temps pourrait être causée par le vieillissement. La figure 2 illustre les raisons pour lesquelles les personnes âgées vivant en milieu rural ou urbain disaient que leurs besoins de soins de santé n'avaient pas été satisfaits pour la période de 1994 à 2004 . Parmi les raisons les plus courantes qui expliquent que les besoins en soins de santé des personnes âgées vivant en milieu rural ne sont pas satisfaits, on note la difficulté d'accès (40 \% des répondants) et la volonté de ne pas consulter un professionnel de la santé (25\% des répondants). Pour leurs homologues vivant en milieu urbain, la raison la plus courante servant à expliquer l'insuffisance de soins de santé pour les personnes âgées était la difficulté d'accès à des professionnels de la santé $(20 \%$ des répondants); d'autres raisons comptaient pour $45 \%$ des réponses, et ce sont les suivantes : trop occupé, ne m’en suis pas encore occupé, ne savais pas où m'adresser, problèmes de transport, problèmes de langue, et obligations personnelles ou familiales. Il est intéressant de noter que tant les personnes âgées vivant en milieu rural que celles vivant en milieu urbain, soit $33 \%$ et $26 \%$ respectivement, qui ont rapporté un niveau de détresse modéré ou élevé avaient consulté relativement souvent (soit plus de 6 fois au cours des douze derniers mois) leur médecin de famille. Les participants en milieu rural avaient moins tendance à consulter leur médecin de famille que leurs homologues vivant en milieu urbain (18\% contre $14 \%$ ).

\section{Résultats de l'analyse à une variable}

Des analyses ont été effectuées afin d'examiner le lien entre l'échelle de détresse, les principaux facteurs considérés et les variables démographiques et socioéconomiques mentionnées ci-dessus.
L’analyse préliminaire a montré que les variables sexe, niveau de scolarité, groupe d'âge, situation familiale, niveau de revenu, état de santé général, zone géographique, usage du tabac, condition chronique, activité physique, autodéclaration de besoins de soins de santé non satisfaits et lieu de résidence étaient liées à l'échelle de détresse psychologique à un niveau de signification de $\alpha=0,20$. La consommation d'alcool n'est pas apparue comme un risque de détresse psychologique dans l'analyse préliminaire et n'a donc pas été utilisée dans le modèle.

\section{Résultats de l'analyse à plusieurs variables}

Le Tableau 2 explique les quatre schémas de données manquantes. Nous pouvons comparer les répondants qui ont terminé l'étude, soit ceux qui ont complété les six cycles, avec ceux qui ont manqué un cycle et ceux qui ont manqué deux cycles ou plus. Nous pouvons aussi comparer les répondants qui ont complété l'étude avec ceux qui sont décédés au cours des six cycles.

Nous avons inclus les schémas de données manquantes et les covariables dans le modèle à plusieurs variables. Cette analyse multivariée est fondée sur la méthode 
FIGURE 2

Raisons pour lesquelles les soins de santé ne sont pas reçus en milieu rural et en milieu urbain

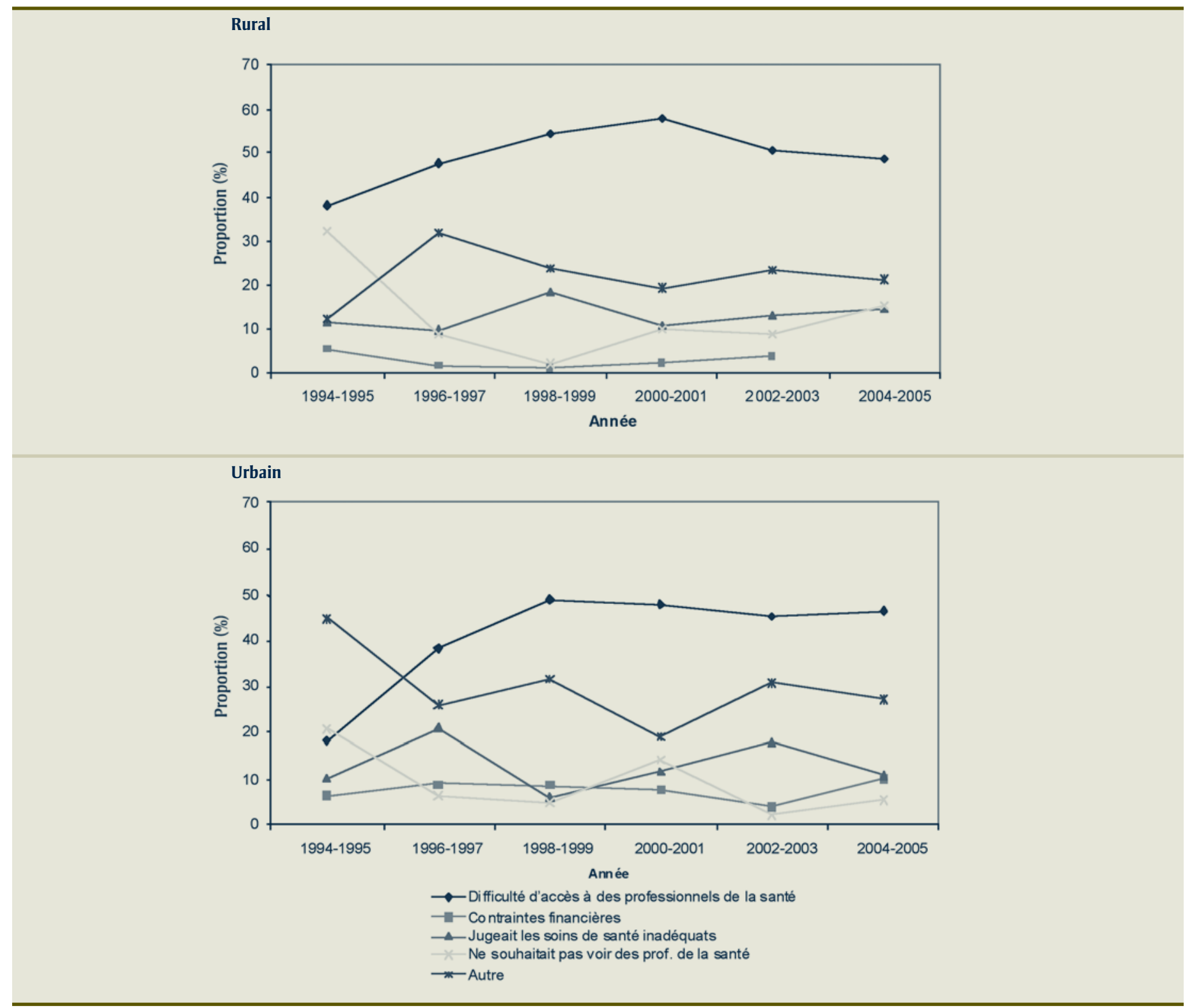

des équations d'estimation généralisées, et les résultats pertinents sont présentés au Tableau 3. Ce modèle est appelé modèle de mélange de schémas fondé sur l’EEG. Dans cette analyse, plusieurs termes d'interaction importants ont été testés (sexe et usage du tabac, sexe et activité physique, etc.) et aucun ne s'est avéré significatif.

Les variables âge, sexe, situation familiale, lieu de résidence, zone géographique, niveau de revenu, niveau de scolarité, usage du tabac, état de santé général, condition chronique, activité physique au cours des trois derniers mois et autodéclaration de besoins de soins de santé non satisfaits ont été incluses dans le modèle aux fins de l'application de la méthode des équations d’estimation généralisées.

Toutes ces variables ont été retenues pour le modèle final qui sert à établir le rapport entre les covariables et la détresse psychologique chez les personnes âgées de 55 ans et plus. Les rapports de cotes indiqués pour toutes les covariables prédisant la détresse psychologique ont pris en compte le lien entre chacune de ces variables et chaque résultat à chaque cycle. Tous les rapports de cotes indiqués ont été corrigés en fonction de toutes les autres variables du modèle. Les rapports de cotes expriment la probabilité que les personnes qui ont une perception négative de leur état de santé général présentent une plus grande détresse psychologique que les personnes qui qualifient leur état de santé général d'excellent. Ce rapport prend en compte l'évolution de la perception qu'ont les personnes de leur état de santé général au cours du suivi de deux ans, afin d'obtenir une estimation globale de l'association 
TABLEAU 1

Données démographiques de base et autre information suivant le lieu de résidence pour l'année 1994

\begin{tabular}{|c|c|c|c|}
\hline & $\begin{array}{c}\text { Degré de détresse psychologique } \\
\text { (modéré/élevé) - participants } \\
\text { en milieu rural }\end{array}$ & $\begin{array}{c}\text { Degré de détresse psychologique } \\
\text { (modéré/élevé) - participants } \\
\text { en milieu urbain }\end{array}$ & Valeur $p$ \\
\hline \multicolumn{4}{|l|}{ Données démographiques } \\
\hline \multicolumn{4}{|l|}{ Groupe d'âge } \\
\hline 55 à 59 & 19,1 & 15,7 & $<0,0001$ \\
\hline 60 à 64 & 13,5 & 17,4 & $<0,0001$ \\
\hline 65 à 69 & 16,6 & 14,8 & $<0,0001$ \\
\hline 70 à 74 & 12,5 & 16,3 & $<0,0001$ \\
\hline 75 à 79 & 24,6 & 13,8 & $<0,0001$ \\
\hline 80 ans et plus & 18,2 & 19,4 & 0,1579 \\
\hline \multicolumn{4}{|l|}{ Sexe } \\
\hline Homme & 11,8 & 11,0 & $<0,0001$ \\
\hline Femme & 22,2 & 19,8 & $<0,0001$ \\
\hline \multicolumn{4}{|l|}{ Situation familiale } \\
\hline Marié/conjoint de fait/partenaire & 17,1 & 13,7 & $<0,0001$ \\
\hline Séparé/veuvage/divorcé & 14,5 & 21,6 & $<0,0001$ \\
\hline Célibataire & 22,6 & 13,9 & $<0,0001$ \\
\hline \multicolumn{4}{|l|}{ Zone géographique } \\
\hline Atlantique & 19,8 & 12,0 & $<0,0001$ \\
\hline Québec & 24,7 & 23,0 & $<0,0001$ \\
\hline Ontario & 13,1 & 14,4 & $<0,0001$ \\
\hline Prairies & 14,6 & 14,9 & 0,0039 \\
\hline Colombie-Britannique & 10,2 & 11,3 & $<0,0001$ \\
\hline \multicolumn{4}{|l|}{ Données socioéconomiques } \\
\hline \multicolumn{4}{|l|}{ Niveau de scolarité } \\
\hline Études secondaires non complétées & 19,1 & 22,5 & $<0,0001$ \\
\hline Diplôme d'études secondaires & 13,9 & 13,1 & $<0,0001$ \\
\hline Études postsecondaires partielles & 13,8 & 10,5 & $<0,0001$ \\
\hline Diplôme d'études postsecondaires & 13,5 & 11,1 & $<0,0001$ \\
\hline \multicolumn{4}{|l|}{ Niveau de revenu } \\
\hline Faible & 22,7 & 22,7 & 1,0000 \\
\hline Moyen & 16,6 & 14,8 & $<0,0001$ \\
\hline Élevé & 4,4 & 10,7 & $<0,0001$ \\
\hline \multicolumn{4}{|l|}{ Soutien social } \\
\hline \multicolumn{4}{|l|}{ Score pour la participation à la vie sociale } \\
\hline Faible & 19,6 & 19,6 & 1,0000 \\
\hline Modéré & 16,4 & 16,8 & $<0,0001$ \\
\hline Élevé & 15,1 & 11,4 & $<0,0001$ \\
\hline \multicolumn{4}{|l|}{ Habitudes de vie } \\
\hline \multicolumn{4}{|l|}{ Usage du tabac } \\
\hline Fumeur & 19,2 & 20,5 & $<0,0001$ \\
\hline Ex-fumeur & 15,5 & 15,5 & 1,0000 \\
\hline Non-fumeur & 17,4 & 14,7 & $<0,0001$ \\
\hline
\end{tabular}


TABLEAU 1 (suite)

Données démographiques de base et autre information suivant le lieu de résidence pour l'année 1994

\begin{tabular}{|c|c|c|c|}
\hline & $\begin{array}{c}\text { Degré de détresse psychologique } \\
\text { (modéré/élevé) - participants } \\
\text { en milieu rural }\end{array}$ & $\begin{array}{c}\text { Degré de détresse psychologique } \\
\text { (modéré/élevé) - participants } \\
\text { en milieu urbain }\end{array}$ & Valeur $p$ \\
\hline \multicolumn{4}{|l|}{ Consommation d'alcool } \\
\hline Consomme de l'alcool & 15,5 & 13,7 & $<0,0001$ \\
\hline Ne consomme plus d'alcool & 19,1 & 21,3 & $<0,0001$ \\
\hline Non-consommateur & 21,3 & 21,2 & 0,4252 \\
\hline \multicolumn{4}{|l|}{ État de santé général } \\
\hline Mauvais & 65,1 & 58,9 & $<0,0001$ \\
\hline Passable & 32,0 & 33,5 & $<0,0001$ \\
\hline Bon & 13,6 & 14,5 & $<0,0001$ \\
\hline Très bon & 6,4 & 6,2 & $<0,0001$ \\
\hline Non & 10,9 & 7,6 & $<0,0001$ \\
\hline \multicolumn{4}{|l|}{ Activité physique au cours des trois derniers mois } \\
\hline Oui & 14,3 & 14,7 & $<0,0001$ \\
\hline Non & 31,5 & 24,1 & $<0,0001$ \\
\hline \multicolumn{4}{|c|}{ Autodéclaration de besoins de soins de santé non satisfaits } \\
\hline Oui & 42,5 & 48,9 & $<0,0001$ \\
\hline Non & 16,2 & 15,0 & $<0,0001$ \\
\hline \multicolumn{4}{|l|}{ Raisons pour ne pas recevoir de soins de santé $\dagger$} \\
\hline Difficulté d'accès à des professionnels de la santé & 47,2 & 61,5 & \\
\hline Aucune & 11,9 & 8,2 & $<0,0001$ \\
\hline 1 à 6 fois & 13,4 & 15,1 & $<0,0001$ \\
\hline Plus de 6 fois & 32,8 & 25,8 & $<0,0001$ \\
\hline
\end{tabular}

$F^{*}$ - Pour des raisons de confidentialité, les petits pourcentages ne sont pas indiqués.

† Valeur $p$ non indiquée

TABLEAU 2

Schéma des données manquantes sur la détresse psychologique durant les six cycles

\begin{tabular}{lc}
\hline Description & Pourcentage \\
\hline Complété les six cycles & 38,43 \\
Un cycle manquant & 11,87 \\
Deux cycles manquants ou plus & 20,80 \\
Personnes décédées au cours des six cycles & 28,90 \\
\hline
\end{tabular}


TABLEAU 3

Rapports de cotes (RC) et intervalles de confiance à $95 \%$ (IC à $95 \%$ ), selon les résultats de la régression logistique binaire multidimensionnelle (modèle de mélange de schémas fondé sur l'EEG) du degré de détresse psychologique

\begin{tabular}{|c|c|c|}
\hline & & RC (IC à $95 \%)$ \\
\hline Non-réponse : & Un cycle manquant & $1,44(1,13,1,82)$ \\
\hline & Deux cycles manquants ou plus & $1,39(1,10,1,76)$ \\
\hline & Décédé au cours des six cycles & $1,68(1,36,2,06)$ \\
\hline & Complété les six cycles & Référence \\
\hline Groupe d'âge : & : 55 à 59 & $1,76(1,31,2,35)$ \\
\hline & 60 à 64 & $1,49(1,13,1,97)$ \\
\hline & 65 à 69 & $1,21(0,93,1,59)$ \\
\hline & 70 à 74 & $1,07(0,84,1,38)$ \\
\hline & 75 à 79 & $1,01(0,80,1,27)$ \\
\hline & 80 et plus & Référence \\
\hline Sexe : & Femme & $1,79(1,47,2,17)$ \\
\hline & Homme & Référence \\
\hline Situation fami & iliale & \\
\hline Marié/conjoi & int de fait/partenariat & $1,19(0,87,1,64)$ \\
\hline Séparé/veuva & goge/divorcé & $1,48(1,08,2,03)$ \\
\hline Célibataire & & Référence \\
\hline Lieu de résiden & & \\
\hline Rural & & $1,16(0,98,1,37)$ \\
\hline Urbain & & Référence \\
\hline Zone géograph & hique & \\
\hline Atlantique & & $0,68(0,53,0,87)$ \\
\hline Québec & & $1,57(1,20,2,06)$ \\
\hline Ontario & & Référence \\
\hline Prairies & & $1,00(0,80,1,25)$ \\
\hline Colombie-Bri & itannique & $0,87(0,67,1,13)$ \\
\hline Statut socioéc & conomique & \\
\hline Niveau de scol & larité & \\
\hline Études secon & Idaires non complétées & $1,36(1,07,1,72)$ \\
\hline Diplôme d'ét & tudes secondaires & $1,28(0,94,1,73)$ \\
\hline Études postse & econdaires partielles & $1,11(0,84,1,47)$ \\
\hline Diplôme d'ét & tudes postsecondaires & Référence \\
\hline Niveau de reve & & \\
\hline Faible & & $1,30(0,89,1,89)$ \\
\hline Moyen & & $1,20(0,88,1,64)$ \\
\hline Élevé & & Référence \\
\hline Habitudes de & & \\
\hline Usage du tabac & & \\
\hline Fumeur & & $1,26(1,00,1,60)$ \\
\hline Ex-fumeur & & $1,10(0,92,1,31)$ \\
\hline Non-fumeur & & Référence \\
\hline
\end{tabular}

pour chaque lien. Une interprétation similaire peut être appliquée à chacune des autres variables du modèle de la détresse psychologique.

Les résultats suivants ont été obtenus à partir du modèle final à plusieurs variables employé pour étudier le rapport entre le lieu de résidence, l'usage du tabac et la détresse psychologique, en y apportant les corrections qui tiennent compte d'autres covariables importantes et du schéma des données manquantes. Les personnes âgées de 55 ans et plus vivant en milieu rural font état d'une plus grande détresse psychologique $[\mathrm{RC}=1,16$; IC à $95 \%$ $(0,98$ à 1,37)] que les personnes âgées vivant en milieu urbain. Après avoir effectué les corrections en fonction des autres covariables $[\mathrm{RC}=1,26$; IC à $95 \%$ $(1,00$ à 1,60$)]$, on observe une association significative évidente entre les personnes âgées qui éprouvent des problèmes de santé mentale et l'usage du tabac. Les personnes âgées qui n'ont pas complété les six cycles étaient significativement plus nombreuses à faire état de détresse psychologique que celles qui les ont complétés [RC $=1,44$ $(1,13$ à 1,82$), \mathrm{RC}=1,39(1,10$ à 1,76$)$ et $\mathrm{RC}=1,68(1,36$ à 2,06$)$ respectivement]. Les participantes féminines étaient significativement plus nombreuses que les participants masculins $[\mathrm{RC}=1,79(1,47$ à 2,17)] à faire état de détresse psychologique. Les participants séparés, veufs ou divorcés ont rapporté un niveau de détresse psychologique significativement plus élevé que les participants célibataires $[\mathrm{RC}=1,48$ $(1,08$ à 2,03)]. Les participants du Québec étaient significativement plus nombreux à faire état de détresse psychologique que les participants de l'Ontario [ $\mathrm{RC}=1,57$ $(1,20$ à 2,06$)]$, tandis que les résidents de l'Atlantique étaient moins nombreux à être dans la détresse que les participants de l'Ontario $[\mathrm{RC}=0,68(0,53$ à 0,87$)]$. Les personnes âgées participantes qui n'ont pas terminé leur cours secondaire étaient plus exposées au risque de détresse psychologique $[\mathrm{RC}=1,36(1,07$ à 1,72)] que les participants qui détenaient un diplôme d'études postsecondaires. Les participants qui qualifiaient leur état de santé général de " mauvais ", " passable " ou "bon " étaient plus exposés au risque de détresse psychologique que ceux qui qualifiaient 
TABLEAU 3 (suite)

Rapports de cotes (RC) et intervalles de confiance à $95 \%$ (IC à $95 \%$ ), selon les résultats de la régression logistique binaire multidimensionnelle (modèle de mélangé de schémas fondé sur l'EEG) du degré de détresse psychologique

\begin{tabular}{ll}
\hline & RC (IC à 95 \%) \\
\hline Santé & \\
État de santé général & $12,14(7,69,19,18)$ \\
Mauvais & $5,26(3,51,7,88)$ \\
Passable & $2,74(1,85,4,06)$ \\
Bon & $1,31(0,89,1,95)$ \\
Très bon & Référence \\
Excellent & \\
Condition chronique* & $1,60(1,29,1,99)$ \\
Oui & Référence \\
Non & \\
Activité physique au cours des trois derniers mois & $0,82(0,70,0,95)$ \\
Oui & Référence \\
\hline Non & \\
\hline Autodéclaration de besoins de soins de santé non satisfaits & $1,72(1,38,2,13)$ \\
Oui & Référence \\
\hline Non & \\
\hline
\end{tabular}

* Une ou plusieurs conditions chroniques

leur état de santé général "d'excellent ". Les personnes âgées participantes qui avaient une condition chronique étaient plus nombreuses à souffrir de détresse psychologique $[\mathrm{RC}=1,60(1,29$ à 1,99$)]$ que celles qui n'avaient pas de condition chronique. Les participants qui avaient fait de l'activité physique au cours des trois derniers mois étaient moins exposés au risque de détresse psychologique $[R C=0,82(0,70$ à 0,95$)]$ que ceux qui n'en avaient pas fait. Pour plusieurs raisons, les participants qui avaient déclaré des besoins de soins de santé non satisfaits étaient relativement plus nombreux à souffrir de détresse psychologique que ceux qui avaient dit recevoir des soins de santé suffisants $[\mathrm{RC}=1,72(1,38$ à 2,13$)]$. La difficulté d'avoir accès à des professionnels de la santé était la principale raison invoquée par une majorité de répondants pour expliquer que leurs besoins de soins de santé n'étaient pas satisfaits.

Nous présentons ici le modèle corrigé en fonction du schéma des valeurs manquantes. Que l'on procède ou non à une correction en fonction des valeurs manquantes, le modèle présente de légères différences dans les estimations des paramètres, les erreurs-types et les RC. Par conséquent, un modèle de mélange de schémas fondé sur l'EEG permet de gommer cet écart dû aux valeurs manquantes.

\section{Analyse}

Il n'y a pas eu à ce jour de véritable analyse statistique de la santé mentale des personnes âgées au Canada qui tienne compte à la fois de la complexité des données longitudinales de l'ENSP (à savoir les six cycles) et de la nature hiérarchique du plan de sondage complexe à plusieurs degrés. De plus, cette analyse a été corrigée pour tenir compte des valeurs manquantes concernant la variable étudiée, ce qui éliminera le biais contenu dans les estimations. Nous avons relevé des différences significatives pour les caractéristiques de base (à savoir le sexe, la situation familiale, la zone géographique, le niveau d'instruction, le niveau de revenu, le soutien social, l'usage du tabac, la consommation d'alcool, l'état de santé général, l'activité physique et l'autodéclaration de besoins de soins de santé non satisfaits) et pour le lieu de résidence (urbain ou rural).
Nous avons observé que les personnes âgées vivant en milieu rural font plus souvent état d'une détresse psychologique que celles vivant en milieu urbain. En outre, il y a une association à long terme significative entre l'usage du tabac et la détresse psychologique. Nos résultats ont démontré qu'il y avait une association à long terme significative entre l'autodéclaration de l'insuffisance de soins de santé et la santé mentale tant chez les personnes âgées ( 55 ans et plus) vivant en milieu rural que chez celles vivant en milieu urbain.

La plupart des études portant sur la santé mentale et qui utilisent des ensembles de données longitudinales comme celles provenant de l'ENSP ont pour thème la dépression. Dans notre étude, nous nous intéressons plutôt à la détresse psychologique. Certaines conclusions de notre étude rejoignent les conclusions de ces études. L'étude réalisée par Stephens et al. ${ }^{22}$ rapporte qu'il n'y a pas de lien entre la santé mentale et des revenus adéquats; nos résultats nous ont aussi mené à cette conclusion. Toujours selon Stephens et al. ${ }^{23}$, les problèmes de santé physique et mentale sont reliés. Nous avons également observé une association entre les répondants faisant mention d'un état de santé général frêle et la détresse psychologique. Nos observations faisant état d'un meilleur état de santé mentale chez les hommes que chez les femmes rejoignent les conclusions de Stephens et al. ${ }^{23}$ et de Østbye et al. ${ }^{35}$ Tout comme Stephens et al. ${ }^{23}$, nous avons observé que les problèmes de santé physique chroniques étaient étroitement associés à la santé mentale.

Contrairement aux conclusions de Stephens et al. ${ }^{23}$ sur la province de résidence, nous avons trouvé qu'il y a une différence significative entre les niveaux de détresse psychologique qu'éprouvent les personnes âgées dans certaines zones géographiques. Les participants du Québec sont proportionnellement plus nombreux que les participants de l'Ontario à rapporter un niveau de détresse psychologique élevé, tandis que les résidants de l'Atlantique sont proportionnellement moins nombreux que ceux de l'Ontario à rapporter un niveau de détresse psychologique élevé. 
Il y a une possibilité de causalité inverse, tel que le démontrent d'autres chercheurs ${ }^{36-40}$. Murphy et al. ${ }^{36}$ ont rapporté que l'usage du tabac en soi n'était pas relié à une manifestation subséquente de la dépression. De plus, ils ont conclu que les participants qui deviennent dépressifs ont plus de chances de commencer ou de continuer à fumer que les participants qui ne sont jamais dépressifs. Lasser et al. ${ }^{39}$ ont rapporté que les personnes qui ont des problèmes de santé mentale ont environ deux fois plus de chances de fumer. Saffer et $a l .{ }^{40}$ ont conclu que les personnes qui ont des antécédents de santé mentale déficiente ont $94 \%$ plus de chances de fumer que les personnes qui ne présentent pas de tels antécédents. Cette étude avait pour objet d'examiner l'association à long terme entre la santé mentale et l'usage du tabac. Afin de déterminer le sens du lien de causalité, il faut procéder à une analyse spéciale. L'ENSP mesure l'autodéclaration de besoins de soins de santé non satisfaits en posant la question suivante : " $\mathrm{Au}$ cours des 12 derniers mois, y a-t-il eu un moment où vous avez cru que vous aviez besoin de soins de santé mais vous ne les avez pas reçus? » La réponse " oui » était enregistrée comme un besoin non satisfait. En raison du libellé de la question portant sur les besoins non satisfaits, il n'est pas possible de distinguer les situations où les personnes n'ont pas reçu de services du tout et celles où elles ne les ont pas reçus en temps opportun. Chen et al. ${ }^{41}$ (2002) et Sanmartin et al. ${ }^{42}$ (2002) ont rapporté que les individus ayant une condition chronique, y compris ceux qui éprouvent de la douleur ou de la détresse, avaient plus tendance à rapporter des problèmes avec le système de distribution des soins médicaux. Plusieurs études ${ }^{43}$ portant sur la santé des personnes âgées ont conclu que le système de santé n'améliore que de façon minime l'état de santé général des personnes âgées. Nos résultats, qui rejoignent les conclusions des études mentionnées ci-dessus, pointent vers une possible causalité inverse. En ce sens, les besoins non satisfaits sont l'effet de la détresse et non la cause. Afin de déterminer le sens de la causalité, il faut procéder à une analyse plus poussée.
Ces résultats peuvent être utilisés pour améliorer la conception et la distribution des soins de santé mentale aux personnes âgées vivant en milieu urbain et en milieu rural. Les résultats peuvent également être employés pour concevoir des méthodes qui réduiront l'usage du tabac chez les personnes âgées vivant en milieu rural et en milieu urbain et pour supprimer les causes de la non-satisfaction des besoins de soins de santé. Une meilleure conception et une meilleure distribution des services peuvent entraîner une réduction des coûts en ce qui a trait à la perte de productivité et au nombre de personnes âgées ayant recours à la psychothérapie, aux visites des salles d'urgence et à la médication.

\section{Remerciements}

Nous désirons remercier Santé publique et agroécosystème en milieu rural (PHARE), le programme des IRSC, pour avoir financé cette étude, de même que le service d'accès aux données à distance de Statistique Canada. Nous voulons remercier tout particulièrement $\mathrm{D}^{\mathrm{re}}$ Lilian Thorpe, gérontopsychiatre, pour ses commentaires judicieux qui ont contribué à améliorer cette étude.

\section{Références}

1. Agence de la santé publique du Canada. Quelques statistiques sur les aînés canadiens - $\mathrm{N}^{\circ} 1$ - $\mathrm{N}^{\circ} 37$ [Internet], Ottawa, Agence de la santé publique du Canada, 2005. Disponible de : http://www.phacaspc.gc.ca/seniors-aines/pubs/factoids/ 2001/toc_f.htm

2. BC Partners for Mental Health and Addictions Information. The primer: facts sheets on mental health and addictions issues [Internet], Colombie-Britannique, Association canadienne pour la santé mental - Division de la Colombie-Britannique, 2003. 127 p. Disponible de : www.bcss.org/ documents/primer.pdf

3. Stotts RC, Smith CK. Smoking patterns among rural elderly [Internet]. South J Nurs Res. 2002; 3(4):1-14. Disponible de : http://www.snrs.org/publications/SOJNR_ articles/iss04vol03.htm\#inter
4. Spencer C. Older adults, alcohol and depression [Internet]. National project report: seeking solutions: Canadian community action on seniors and alcohol issues, Vancouver, Gerontology Research Centre, Simon Fraser University, 2003 mai. Disponible de : http://www.agingincanada. ca/Alcohol and Depression_7.pdf

5. Dewa CS, Lesage A, Goering P, Caveen M. Nature and prevalence of mental illness in the work place. Healthc Pap. 2004; 5(2):12-25.

6. Stephens T, Joubert N. Le fardeau économique des problèmes de santé mentale au Canada. Maladies chroniques au Canada. 2001;22(1):19-25.

7. Murray CJL, Lopez AD, (dir.). The global burden of disease: A comprehensive assessment of mortality and disability from diseases, injuries, and risk factors in 1990 and projected to 2020. Vol. 1, Cambridge (MA), Harvard University Press, 1996. 990 p.

8. Organisation mondiale de la Santé. Rapport sur la santé dans le monde, 2001 - La santé mentale : Nouvelle conception, nouveaux espoirs, Genève, Organisaiton mondiale de la Santé, 2001. 178 p.

9. Stephens T, Joubert N. The economic burden of mental health problems in Canada. Chronic Dis Can. 2001; 22(1):18-23.

10. Statistique Canada. Enquête nationale sur la santé de la population - volet ménages, cycle 6 (2004/2005): documentation longitudinale [Internet], Ottawa, Statistique Canada, 2006. Disponible de : http://www. statcan.gc.ca/imdb-bmdi/document/3225_ D5_T1_V3-fra.pdf

11. Statistique Canada. Enquête nationale sur la santé de la population - volet ménages, cycle 6 (2004/2005): documentation des variables dérivées et des variables longitudinales constantes [Internet], Ottawa, Statistique Canada, 2006. Disponible de : http://www.statcan.gc.ca/imdb-bmdi/ document/3225_D10_T9_V2-fra.pdf

12. Statistique Canada. Fichier de microdonnées à grande diffusion : enquête nationale sur la santé de la population - 1994-1995, Ottawa, Statistique Canada, 1995. 64 p. 
13. Bellerose C, Lavallée C, Tremblay D. Cahier technique et méthodologique. Enquête sociale et de santé 1992-1993.Vol. 1, Montréal, Gouvernement du Québec, Ministère de la Santé et des Services sociaux, 1995. $134 \mathrm{p}$.

14. Buckley NJ, Denton FT, Robb AL, Spencer BG. Socio-economic influence on the health of older people: estimates based on two longitudinal surveys, Hamilton, Research Institute for Quantitative Studies in Economics and Population (QSEP), 2003. Rapport $\mathrm{n}^{\circ} 387$.

15. Michiels B, Molenberghs GM, Bijnens L, Vangenengden T, Thijs H. Selection models and pattern mixture models to analyze longitudinal quality of life data subject to dropout. Stat Med. 2002;21:1023-41.

16. Little RJ, Rubin DB. Statistical analysis with missing data. Chapitres 14 et 15, New York, John Wiley and Sons, 2002. p. 292-348.

17. Little RJ. Pattern - mixture models for multivariate incomplete data. J Am Stat Assoc. 1993; 88:125-34.

18. Little RJ. A class of pattern-mixture models for normal missing data. Biometrika. 1994; 81:471-83.

19. Kessler R, Mroczek D. Final versions of our non-specific psychological distress scale, Ann Arbor (MI), Survey Research Centre of the Institute for Social Research, University of Michigan. La note de service date du 10 mars 1994.

20. Patten SC, Beck CA. Major depression and mental health care utilization in Canada: 1994-2000. Can J Psychiatry. 2004; 49(5):303-9.

21. Wang J, El-Guebaly N. Sociodemographic factors associated with comorbid major depression episodes and alcohol dependence in the general population. Can J Psychiatry. 2004 Jan; 49(1):37-44.

22. Wilkins K, Beaudet MP. Work stress and health. Health Rep. 1998;10(3):47-62.

23. Stephens T, Dulberg C, Joubert N. Mental health of the Canadian population: a comprehensive analysis. Chronic Dis Can. 2000;20(3):118-26.

24. Baggaley RF, Ganaba R, Fillippi V, Kere M, Marshall T, Sombie I, Storeng KT, Patel V. Detecting depression after pregnancy: the validity of the K10 and K6 in Burkina Faso. Trop Med Int Health. 2007;12(10):1225-9.

25. Allison, PD. Logistic regression using SAS: theory and application, Cary (NC), SAS Institute, 1999. p. 5-78, 179-213.

26. SAS Institute Inc. SAS/STAT 9.1 user's guide, Cary (NC), SAS Institute Inc., 2005. 5136 p. Disponible de : http://support.sas. com/documentation/onlinedoc/91pdf/ index_913.html

27. Diggle PJ, Liang K-Y, Zeger SL. Analysis of longitudinal data, New York, Oxford University Press, 1994. 253 p.

28. Liang K-Y, Zeger SL. Longitudinal data analysis using generalized estimating equations. Biometrika. 1986;73:13-22.

29. Zeger SL, Liang K-Y. Longitudinal data analysis for discrete and continuous outcomes. Biometrics. 1986;42:121-30.

30. SAS Institute Inc. Longitudinal data analysis with discrete and continuous responses: instructor based training, Cary (NC), SAS Institute Inc., 2002. p. 3-32.

31. Statistique Canada. Estimation of the variance using the bootstrap weights. User's guide for the BOOTVARE_V21.SPS program. Version 2.1, Ottawa, Statistique Canada, 2005.

32. Rao JN. Interplay between sample survey theory and practice: an appraisal. Surv Methodol. 2005; 31(2):117-38.

33. Binder DA, Roberts GR. Statistical inference in survey data analysis: where does the sample design fit in? Manuscrit présenté au : Statistics Canada Research Data Centre Conference Program, University of McMaster, 2003, 24-25 sept., Hamilton (ON). Disponible de : http://socserv.socsci. mcmaster.ca/rdc2003/binderoberts.pdf
34. Fleming SA, Bains N, Hunter DJ, Lam M. Social support and health care use among a sample of healthy Canadians: a longitudinal analysis of the national population health survey. Kingston (ON), Health information partnership, Eastern Ontario Region, 2004. 58 p.

35. Østbye T, Kristjansson B, Hill G, Newman SC, Brouwer RN, NcDowell I. Étude sur la santé et le vieillissement au Canada : prévalence et prédicteurs de la dépression chez les personnes âgées au Canada. Maladies chroniques au Canada. 2005;26(4):101-08.

36. Murphy JM, Horton NJ, Monson RR, Laird NM, Sobol AM, Leighton AH. Cigarette smoking in relation to depression: historical trends from the Stirling country study. Am J Psychiatry. 2003;160:1663-9.

37. Anda RF, Williamson DF, Escobedo LG, Mast EE, Giovino GA, Remington PL. Depression and the dynamics of smoking: a national perspective. JAMA. 1990;264(12): 1541-5.

38. Hughes JR, Hatsukami DK, Mitchell JE, Dahlgren LA. Prevalence of smoking among psychiatric outpatients. Am J Psychiatry. 1986;143:993-7.

39. Lasser K, Boyd JW, Woolhandler S, Himmelstein DU, McCormick D, Bor DH. Smoking and mental illness: a populationbased prevalence study. JAMA. 2000; 284(20):2606-10.

40. Saffer H, Dave D. Mental illness and the demand for alcohol, cocaine and cigarettes. Economic Inquiry, Oxford University Press, 2005 April; Vol. 43(2): 229-246.

41. Chen J, Hou F. Unmet needs for health care. Rapports sur la santé. 2002;13(2)27-40. $\mathrm{N}^{\circ}$ au catalogue : 82-003-XIE.

42. Sanmartin C, Houle C, Tremblay S, Berthelot JM. Changes in unmet health care needs. Rapports sur la santé. 2002;13(3):17-24. $\mathrm{N}^{\circ}$ au catalogue : 82-003-XIE.

43. Martin-Matthews A. De la connaissance à la pratique : Le Fonds pour l'adaptation des services de santé : Série de rapports de synthèse, Ottawa, Santé Canada, 2002. $\mathrm{N}^{\circ}$ au catalogue : H13-6/2002-7. 